\title{
EULER-TYPE APPROXIMATION FOR SYSTEMS OF STOCHASTIC DIFFERENTIAL EQUATIONS *
}

\author{
J. Golec \\ Department of Mathematics \\ Fordham University \\ Bronx, New York 10458 \\ and \\ G. Ladde \\ Department of Mathematics \\ The University of Texas at Arlington \\ Arlington, Texas 76019
}

\begin{abstract}
By developing a stochastic version of the Taylor formula, the mean-square convergence of the Euler-type approximation for the solution of systems of Itô-type stochastic differential equations is investigated. Sufficient conditions are given to obtain time-varying and time-invariant error estimates.
\end{abstract}

1980 Mathematics Subject Classification. Primary 60H10. Secondary 41A25.

Key words: stochastic differential equation, approximate solution, error estimate.

* Received: July, 1989; Revised: October, 1989 


\section{Introduction}

We consider general systems of Itô-type stochastic differential equations $[2,3]$ of the following form:

$$
\begin{aligned}
d X_{t} & =a\left(t, X_{t}\right) d t+\sigma\left(t, X_{t}\right) d W_{t} \\
X_{t_{0}} & =X_{0},
\end{aligned}
$$

for $t \geq t_{0}$, where $t_{0}$ is a positive number, $a=a(t, x)$ is an n-dimensional vector, $\sigma=\sigma(t, x)$ is an $n \times m$ matrix $\left(x \in R^{n}\right), W=\left(W_{t}\right)_{t \geq 0}$ is the mdimensional (standard) Wiener Process and $X_{0}$ is an n-dimensional, squareintegrable random vector independent of the increments of $W$ on any interval to the right of $t_{0}$. Throughout this paper we assume without mentioning it specifically that coefficients $a$ and $\sigma$ satisfy the classical conditions $[1,2,3]$ for existence and uniqueness of the solution process of system (1.1)-(1.2).

Let us fix positive numbers $T$ and $h$, for which $T / h$ is an integer. The Euler-type difference scheme [4] for system (1.1)-(1.2) is given by

$$
\begin{aligned}
\bar{X}_{t_{k}+\theta} & =\bar{X}_{t_{k}}+a\left(t_{k}, \bar{X}_{t_{k}}\right) \theta+\sigma\left(t_{k}, \bar{X}_{t_{k}}\right)\left(W_{t_{k}+\theta}-W_{t_{k}}\right) \\
\bar{X}_{t_{0}} & =X_{0}
\end{aligned}
$$

for $\theta \in[0, h]$, where $t_{k}=t_{0}+k h, k=0,1, \ldots, T / h-1$.

In this note we first develop a general version of the stochastic Taylor formula which is valid for the solution process of system (1.1). By applying the stochastic Taylor formula and by assuming at most polynomial-type growth conditions for certain derivatives of the coefficients $a$ and $\sigma$, a uniform estimate for the meansquare error of the Euler-type approximation (1.3)-(1.4) is obtained:

$$
\sup _{t \in\left[t_{0}, t_{0}+T\right]} E\left|X_{t}-\bar{X}_{t}\right|^{2} \leq C h,
$$

where $C$ is a constant depending on $T$. The proof in the above general case admits certain modification. As a result we show that if one adds to the set of above assumptions for the coefficients of system (1.1) some stability-type conditions, then the estimate for the mean-square error in (1.5) becomes independent of the length $T$ of the time-interval.

The Euler-type difference scheme considered for general systems of the form (1.1) has the global first order accuracy in the mean-square sense. Higher order accurate stochastic difference schemes were considered in $[5,6,7]$. The increase in the order of accuracy was achieved at the expense of imposing some algebraical conditions for the coefficients of system (1.1) [5], or by using in the approximation process some new random variables, other than the Wiener Process itself [7]. We note [8] that for general systems of form (1.1) the global first order accuracy in the mean-square sense is the best possible order that one may obtain in the context of Runge-Kutta type stochastic difference schemes. 


\section{Auxiliary Result}

In the following we assume that the coefficients $a$ and $\sigma$ have continuous derivatives of all kinds that will appear in this section. Let $\mathcal{L}$ be the infinitesimal generator of the solution process of system (1.1). We have

$$
\mathcal{L}=\frac{\partial}{\partial t}+\sum_{i=1 / n} a_{i}(t, x) \frac{\partial}{\partial x_{i}}+\sum_{i, j=1 / n, n} \gamma_{i j}(t, x) \frac{\partial^{2}}{\partial x_{i} \partial x_{j}}
$$

where $\gamma=\sigma \sigma^{*}$ is the diffusion matrix of the process $X=\left(X_{t}\right)$ and *-denotes the transpose matrix. For any real numbers $\alpha$ and $\beta(\alpha<\beta)$ and for a natural number $d$ let us denote by $M^{2}\left([\alpha, \beta] ; R^{d}\right)$ the vector space of all adapted, measurable $d$-dimensional random vectors $\left(g_{t}\right)_{t \in[\alpha, \beta]}$, for which $\int_{\alpha}^{\beta} E\left\|g_{t}\right\|^{2} d t$ is finite. For the purpose of the next theorem let us suppose that the initial condition (1.2) is of the form

$$
X_{t_{0}}=x_{0}
$$

where $x_{0}$ is a fixed (nonrandom) $n$-dimensional vector.

Theorem 2.1 Let $h_{0}>0$ and $k$ be a natural number. Let $f \in C^{2 k+2}$ be a real-valued function defined for $t \in\left[t_{0}, t_{0}+h_{0}\right]$ and $x \in R^{n}$. We assume that the function $f$, together with the solution process $X$ of system (1.1)-(2.2) satisfy the following conditions:

(i) $M_{p}=\sup _{s \in\left[t_{0}, t_{0}+h_{0}\right]} E\left(\mathcal{L}^{p} f\left(s, X_{s}\right)\right)^{2}<\infty$, for $p=1, \ldots, k+1$,

(ii) $\left\{\frac{\partial\left(\mathcal{L}^{\prime} \mathcal{f}\right)}{\partial x}\left(s, X_{s}\right)\right\}^{*} \sigma\left(s, X_{s}\right) \in M^{2}\left(\left[t_{0}, t_{0}+h_{0}\right] ; R^{m}\right)$, for $p=0,1, \ldots, k$. Then

$$
\begin{aligned}
E\left\{f\left(t_{0}+h_{0}, X_{t_{0}+h_{0}}\right)\right\}= & f\left(t_{0}, x_{0}\right)+(\mathcal{L} f)\left(t_{0}, x_{0}\right) h_{0}+\ldots+\frac{1}{k !}\left(\mathcal{L}^{k} f\right)\left(t_{0}, x_{0}\right) h_{0}^{k} \\
& +\frac{1}{k !} \int_{0}^{h_{0}}\left(h_{0}-\theta\right)^{k} E\left\{\left(\mathcal{L}^{k+1} f\right)\left(t_{0}+\theta, X_{t_{0}+\theta}\right)\right\} d \theta .
\end{aligned}
$$

Proof. Let $\Phi(h)=E\left\{f\left(t_{0}+h, X_{t_{0}+h}\right)\right\}$ for $h \in\left[0, h_{0}\right]$. Applying Itô formula, we obtain

$$
(d f)\left(t, X_{t}\right)=(\mathcal{L} f)\left(t, X_{t}\right) d t+\left\{\frac{\partial f}{\partial x}\left(t, X_{t}\right)\right\}^{*} \sigma\left(t, X_{t}\right) d W_{t} .
$$

Therefore for any $h \in\left[0, h_{0}\right)$ we can write

$$
\begin{aligned}
\frac{1}{\Delta h}[\Phi(h+\Delta h)-\Phi(h)]= & E\left\{\frac{1}{\Delta h} \int_{t_{0}+h}^{t_{0}+h+\Delta h}(\mathcal{L} f)\left(s, X_{s}\right) d s\right. \\
& +E\left\{\frac{1}{\Delta h} \int_{t_{0}+h}^{t_{0}+h+\Delta h}\left\{\frac{\partial f}{\partial x}\left(s, X_{s}\right)\right\}^{*} \sigma\left(s, X_{s}\right) d W_{s}\right\} \\
= & E\left\{\frac{1}{\Delta h} \int_{t_{0}+h}^{t_{0}+h+\Delta h}(\mathcal{L} f)\left(s, X_{s}\right) d s\right\}
\end{aligned}
$$


(where assumption (ii) for $p=0$ has been used). For a fixed $h \in\left[0, h_{0}\right)$ and for sufficiently small positive $\Delta h$ let us define

$$
\Psi_{\Delta h}=\frac{1}{\Delta h} \int_{t_{0}+h}^{t_{0}+h+\Delta h}(\mathcal{L} f)\left(s, X_{s}\right) d s
$$

Using Schwarz inequality and condition (i) for $p=1$, we obtain

$$
E\left|\Psi_{\Delta h}\right|^{2} \leq \frac{1}{\Delta h} \int_{t_{0}+h}^{t_{0}+h+\Delta h} E\left\{(\mathcal{L} f)\left(s, X_{s}\right)\right\}^{2} d s \leq M_{1}<\infty .
$$

From (2.4) we conclude that the family of random variables $\left\{\Psi_{\Delta h}\right\}$ is equiintegrable and therefore we have

$$
\lim _{\Delta h \rightarrow 0} \frac{1}{\Delta h}[\Phi(h+\Delta h)-\Phi(h)]=E\left\{\lim _{\Delta h \rightarrow 0} \frac{1}{\Delta h} \int_{t_{0}+h}^{t_{0}+h+\Delta h}(\mathcal{L} f)\left(s, X_{s}\right) d s\right\} .
$$

The last equality together with sample-path continuity of the process $\mathrm{X}$ yields

$$
\Phi^{\prime}(h)=E\left\{(\mathcal{L} f)\left(t_{0}+h, X_{t_{0}+h}\right)\right\} .
$$

Proceeding in the same way and using assumptions (i) and (ii), we obtain

$$
\Phi^{(p)}(h)=E\left\{\left(\mathcal{L}^{p} f\right)\left(t_{0}+h, X_{t_{0}+h}\right)\right\},
$$

for $p=2, \ldots, k+1$ and $h \in\left[0, h_{0}\right)$. Finally by applying the regular Taylor formula with integral-type remainder we obtain the desired equality.

Remark 2.1 It is obvious from the proof of Theorem 2.1 that by using Taylor formulae with different types of remainders, one can prove the validity of the corresponding versions of the stochastic Taylor formula.

\section{Time-varying Error Estimate}

In the present section we will need the following technical lemma.

Lemma 3.1 There exists a positive constant $M$ depending only on $T$ such that for any $h \in(0,1]$ (with $T / h$ being a natural number) the following is true:

$$
\max _{k=0,1, \ldots, T / h} E\left|\bar{X}_{t_{k}}\right|^{2} \leq M
$$

where $\bar{X}=\left(\bar{X}_{t}\right)_{t \in\left[t_{0}, t_{0}+T\right]}$ is the stochastic Euler difference scheme given by (1.3) $-(1.4)$. 
Proof. By defining $\epsilon_{k}^{2}=E\left|\bar{X}_{t_{k}}\right|^{2}$ (where $t_{k}=t_{0}+k h$, for $k=0,1, \ldots, T / h$ ) and by using basic properties of the Wiener Process $W$ we easily obtain the following recurrence relation for $\epsilon_{k}^{2}$ :

$$
\begin{aligned}
\epsilon_{k+1}^{2} & \leq\left(1+C_{1} h\right) \epsilon_{k}^{2}+C_{2} h \\
\epsilon_{0}^{2} & =E\left|X_{0}\right|^{2},
\end{aligned}
$$

for $k=0,1, \ldots, T / h-1$, where $C_{1}, C_{2}$ are positive constants depending only on the coefficients of system (1.1). From (3.1)-(3.2) we derive the inequality

$$
\epsilon_{k}^{2} \leq\left(1+C_{1} h\right)^{k} E\left|X_{0}\right|^{2}+\frac{C_{2}}{C_{1}}\left[\left(1+C_{1} h\right)^{k}-1\right],
$$

for $k=0,1, \ldots, T / h$. Now the lemma follows immediately.

In the next theorem it is convenient to use the concept of at most polynomial growth. We recall that a function $f=f(y)$ has at most polynomial growth with exponent $\mu$ (and a constant $L$ ) if for any $y$ :

$$
|f(y)| \leq L\left(1+|y|^{\mu}\right) .
$$

Theorem 3.1 Let us assume that the coefficients of system (1.1) have continuous first order partial derivatives with respect to $t$ and continuous first and second order partial derivatives with respect to $x$. Let us suppose that for any $t \in\left[t_{0}, t_{0}+T\right]$ all these derivatives have at most polynomial growth with a fixed exponent $\mu$ (and a common constant $L$ ). We assume that the initial random variable $X_{0}$ in (1.2) is an element of $\mathcal{L}^{2 \mu+4}(\Omega)$. Then

$$
\sup _{t \in\left[t_{0}, t_{0}+T\right]} E\left|X_{t}-\bar{X}_{t}\right|^{2} \leq C h,
$$

where $C$ is a constant depending only on $T$ (and the coefficients of system (1.1)) and $\bar{X}=\left(\bar{X}_{t}\right)_{t \in\left[t_{0}, t_{0}+T\right]}$ denotes the stochastic Euler scheme defined by (1.3)(1.4), for any $h$ with $T / h$ being an integer.

Proof. First, we note that in view of Lemma 3.1 it is sufficient to prove that

$$
\max _{k=0,1, \ldots, T / h} E\left|X_{t_{k}}-\bar{X}_{t_{k}}\right|^{2} \leq C h .
$$

In order to be able to treat the Wiener Process $W$ as an additional state variable we consider the following augmented system of stochastic differential equations:

$$
\begin{aligned}
& d X_{t}=a\left(t, X_{t}\right) d t+\sigma\left(t, X_{t}\right) d W_{t} ; X_{t_{0}}=X_{0}, \\
& d W_{t}=0 d t+I_{m} d W_{t} ; W_{t_{0}}=W_{t_{0}},
\end{aligned}
$$

where $I_{m}$ denotes $m$-dimensional identity matrix. The solution of system (3.5) is a diffusion process with the infinitesimal generator $\mathcal{L}$, where

$$
\mathcal{L}=\frac{\partial}{\partial t}+\sum_{i=1 / n} a_{i}(t, x) \frac{\partial}{\partial x_{i}}+\frac{1}{2} \sum_{i, j=1 / n, n} \gamma_{i j}(t, x) \frac{\partial^{2}}{\partial x_{i} \partial x_{j}}
$$




$$
+\sum_{i, j=1 / n, m} \sigma_{i j}(t, x) \frac{\partial^{2}}{\partial x_{i} \partial w_{j}}+\frac{1}{2} \sum_{j=1 / m} \frac{\partial^{2}}{\partial w_{j}^{2}} .
$$

(as before, we define $\gamma=\sigma \sigma^{*}$ ).

For any fixed natural number $k$ less than $T / h$ let us define

$$
f_{k}(t, x, w, \bar{x}, \bar{w})=\left|x-\bar{x}-a\left(t_{k}, \bar{x}\right)\left(t-t_{k}\right)-\sigma\left(t_{k}, \bar{x}\right)(w-\bar{w})\right|^{2},
$$

for $t \in R, x, \bar{x} \in R^{n}, w, \bar{w} \in R^{m}$. One can easily compute that

$$
\begin{aligned}
& \left(\mathcal{L} f_{k}\right)(t, x, w, \bar{x}, \bar{w})=2\left[x-\bar{x}-a\left(t_{k}, \bar{x}\right)\left(t-t_{k}\right)-\right. \\
& \left.\quad-\sigma\left(t_{k}, \bar{x}\right)(w-\bar{w})\right]\left[a(t, x)-a\left(t_{k}, \bar{x}\right)\right]+\left|\sigma(t, x)-\sigma\left(t_{k}, \bar{x}\right)\right|^{2},
\end{aligned}
$$

where $f_{k}$ is treated as function of variables $(t, x, w)$ and $\bar{x}, \bar{w}$ are treated as parameters, also the norm of a matrix is defined in terms of the sum of squares of its coefficients and for any $n$-dimensional vectors $A$ and $B$ we denote by $[A][B]$ their scalar product. After rather tedious calculations one also obtains the corresponding formula for $\mathcal{L}^{2} f_{k}$.

Let us define $\epsilon_{k}^{2}=E\left|X_{t_{k}}-\bar{X}_{t_{k}}\right|^{2}$, for $k=0,1, \ldots, T / h$. In order to find a recurrence relation for $\epsilon_{k}^{2}$ we write

$$
\begin{aligned}
\epsilon_{k+1}^{2} & =E\left|X_{t_{k}+h}-\bar{X}_{t_{k}+h}\right|^{2} \\
& =E\left\{E_{t_{k}, X_{t_{k}}, W_{t_{k}}, \bar{X}_{t_{k}}, W_{t_{k}}} f_{k}\left(t_{k}+h, X_{t_{k}+h}, W_{t_{k}+h}, \bar{X}_{t_{k}}, W_{t_{k}}\right)\right\}
\end{aligned}
$$

where $E$ with the subscript denotes conditional expectation with respect to the specified random variables and the time $t_{k}$. We are in a position to apply Theorem 2.1 for the inner conditional expectation in (3.9). In particular, the last two variables $\bar{X}_{t_{k}}$ and $W_{t_{k}}$ are fixed for $t \in\left[t_{k}, t_{k}+h\right]$ and therefore the infinitesimal generator will treat them as parameters. We obtain

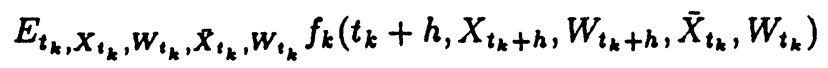

$$
\begin{aligned}
& =f_{k}\left(t_{k}, X_{t_{k}}, W_{t_{k}}, \bar{X}_{t_{k}}, W_{t_{k}}\right)+\left(\mathcal{L} f_{k}\right)\left(t_{k}, X_{t_{k}}, W_{t_{k}}, \bar{X}_{t_{k}}, W_{t_{k}}\right) h \\
& +\int_{0}^{h}(h-\theta) E_{t_{k}, X_{t_{k}}, W_{t_{k}}, \bar{X}_{t_{k}}, W_{t_{k}}}\left\{\left(\mathcal{L}^{2} f_{k}\right)\left(t_{k}+\theta, X_{t_{k}+\theta}, W_{t_{k}+\theta}, \bar{X}_{t_{k}}, W_{t_{k}}\right)\right\} d \theta \\
& =\left|X_{t_{k}}-\bar{X}_{t_{k}}\right|^{2} \\
& +\left\{2\left[X_{t_{k}}-\bar{X}_{t_{k}}\right]\left[a\left(t_{k}, X_{t_{k}}\right)-a\left(t_{k}, \bar{X}_{t_{k}}\right)\right]+\left|\sigma\left(t_{k}, X_{t_{k}}\right)-\sigma\left(t_{k}, \bar{X}_{t_{k}}\right)\right|^{2}\right\} h \\
& +\int_{0}^{h}(h-\theta) E_{t_{k}, X_{t_{k}}, W_{t_{k}}, X_{t_{k}}, W_{t_{k}}}\left\{\left(\mathcal{L}^{2} f_{k}\right)\left(t_{k}+\theta, X_{t_{k}+\theta}, W_{t_{k}+\theta}, \bar{X}_{t_{k}}, W_{t_{k}}\right)\right\} d \theta .
\end{aligned}
$$

By taking expected values on both sides of (3.10) and using assumptions about the coefficients of system (1.1) we obtain:

$$
\epsilon_{k+1}^{2} \leq \epsilon_{k}^{2}+C_{1} h \epsilon_{k}^{2}+\int_{0}^{h}(h-\theta) E\left\{\left(\mathcal{L}^{2} f_{k}\right)\left(t_{k}+\theta, X_{t_{k}+\theta}, W_{t_{k}+\theta}, \bar{X}_{t_{k}}, W_{t_{k}}\right)\right\} d \theta
$$


where $C_{1}$ is a constant depending only on the coefficients of system (1.1). By analyzing the second iteration $\mathcal{L}^{2} f_{k}$, using Lemma 3.1 together with the growth assumptions for $a, \sigma$ and the derivatives we conclude that

$$
E\left\{\left(\mathcal{L}^{2} f_{k}\right)\left(t_{k}+\theta, X_{t_{k}+\theta}, W_{t_{k}+\theta}, \bar{X}_{t_{k}}, W_{t_{k}}\right)\right\} \leq C_{2}<\infty,
$$

for $k=0,1, \ldots, T / h-1, \theta \in[0, h]$, where $C_{2}$ is a constant depending on $T$ and the $\mathcal{L}^{2 \mu+4}$-norm of the initial random variable in (1.2). Inequalities (3.11), (3.12) yield the following recurrence relation for $\epsilon_{k}^{2}$ :

$$
\begin{aligned}
\epsilon_{k+1}^{2} & \leq\left(1+C_{1} h\right) \epsilon_{k}^{2}+C_{2} h^{2} \\
\epsilon_{0}^{2} & =0,
\end{aligned}
$$

for $k=0,1, \ldots, T / h-1$. Relation (3.13)-(3.14) implies that

$$
\epsilon_{k}^{2} \leq \frac{C_{2}}{C_{1}}\left[\left(1+C_{1} h\right)^{T / h}-1\right] h \leq \frac{C_{2}}{C_{1}}\left(e^{C_{1} T}-1\right) h \leq C_{3} h,
$$

for $k=0,1, \ldots, T / h$ and $C_{3}$ is a constant. Finally, (3.15) implies inequality (3.4) and the proof is complete.

It is seen from the inequality (3.15) that the constant $C$ in Theorem 3.1 depends exponentially on the length $T$ of the time-interval. As a result, approximation of the type (1.3)-(1.4) becomes "crude" exponentially fast as the length of the time-interval $T$ increases. In the next section we show a way to remedy this problem.

\section{Time-invariant Error Estimate}

In this section we obtain a result concerning time-invariant estimate for the mean-square error of the Euler-type approximation. First we prove a general lemma that gives a sufficient condition for existence of a time-invariant estimate for higher moments of the solution process.

Lemma 4.1 Let us assume that the coefficients of system (1.1) satisfy the following conditions: $x a(t, x) \leq-\alpha|x|^{2}$ and also $|\sigma(t, x)|^{2} \leq \beta|x|^{2}$, for $t \geq t_{0}$, $x \in R^{n}$, where $\alpha, \beta$ are fixed positive numbers. Let us further assume that for some natural number $p$

$$
E\left|X_{0}\right|^{4 p}<\infty \text { and also } \beta \leq \frac{2 \alpha}{(2 p-1) n} .
$$

Then $E\left|X_{t}\right|^{2 p} \leq \exp \left\{-\gamma\left(t-t_{0}\right)\right\}$ for $t \geq t_{0}$, where $\gamma=2 \alpha p-\beta(2 p-1) n p$. In particular, we have

$$
\sup _{t \in\left[t_{0}, \infty\right)} E\left|X_{t}\right|^{2}<\infty
$$


Proof. We define $f(t, x)=|x|^{2 p} e^{\gamma\left(t-t_{0}\right)}$, for $t \geq t_{0}$ and $x \in R^{n}$. By applying Itô formula for the function $f$ together with the solution process $X$ and by using assumptions we arrive at the following inequality:

$$
\begin{aligned}
\left|X_{t}\right|^{2 p} e^{\gamma\left(t-t_{0}\right)} \leq & \left|X_{0}\right|^{2 p}+\int_{t_{0}}^{t}[\gamma-2 \alpha p+p \beta(2 p-1) n] e^{\gamma\left(s-t_{0}\right)}\left|X_{s}\right|^{2 p} d s \\
& +\int_{t_{0}}^{t} 2 p e^{\gamma\left(s-t_{0}\right)}\left|X_{s}\right|^{2 p-2} X_{s}^{*} \sigma\left(s, X_{s}\right) d W_{s}
\end{aligned}
$$

for $t \geq t_{0}$. Now, taking expected values on both sides of inequality (4.1), using the definition of $\gamma$ and properties of stochastic integral we conclude that

$$
E\left|X_{t}\right|^{2 p} e^{\gamma\left(t-t_{0}\right)} \leq E\left|X_{0}\right|^{2 p} .
$$

The proof is complete.

The next lemma is similar to Lemma 3.1. It establishes an estimate for the second moment of scheme (1.3)-(1.4) that does not depend on the length of the time-interval.

Lemma 4.2 Let us assume that the coefficients of system (1.1) satisfy the following conditions: $x a(t, x) \leq-\alpha|x|^{2}$ and also $|\sigma(t, x)|^{2} \leq \beta|x|^{2}$, for $t \geq t_{0}$, $x \in R^{n}$, where $\alpha, \beta$ are fixed positive numbers. We also assume that $\beta<2 \alpha$. Then there exists $h_{0}>0$ such that for any length $T$ of the time-interval and for any $h \in\left(0, h_{0}\right.$ ] (with $T / h$ being an integer) the following is true:

$$
\max _{k=0,1, \ldots, T / h} E\left|\bar{X}_{t_{k}}\right|^{2} \leq C<\infty .
$$

The constant $C$ is independent of $T$ and $h$ and depends only on the coefficients of system (1.1).

Proof. By recalling the proof of Lemma 3.1 let us observe that under our assumptions the recurrence relation (3.1)-(3.2) becomes

$$
\begin{aligned}
\epsilon_{k+1}^{2} & \leq\left(1-\gamma h+C_{1} h^{2}\right) \epsilon_{k}^{2}+C_{1} h^{2} \\
\epsilon_{0}^{2} & =E\left|X_{0}\right|^{2},
\end{aligned}
$$

for $k=0,1, \ldots, T / h-1$ where $\gamma=2 \alpha-\beta$ and $C_{1}$ is a positive constant independent of $T$. From (4.2)-(4.3) it follows that

$$
\epsilon_{k}^{2} \leq\left(1-\gamma h+C_{1} h^{2}\right)^{k} E\left|X_{0}\right|^{2}+C_{1} h \frac{1-\left(1-\gamma h+C_{1} h^{2}\right)^{k}}{\gamma-C_{1} h},
$$

for $k=Q, 1, \ldots, T / h$. Now, using inequality (4.4) we easily verify that the assertion of the lemma remains valid with $h_{0}=\min \left(\gamma / 2 C_{1}, 1 / \gamma\right)$. The proof is complete.

We are ready to present the main theorem of this section. 
Theorem 4.1 Let us suppose that system (1.1) admits the trivial zero solution. We assume that the same requirements as in Theorem 9.1 with regard to the partial derivatives of $a$ and $\sigma$ are valid. Let us further assume that

$$
\begin{aligned}
|\sigma(t, x)-\sigma(t, y)|^{2} & \leq \beta|x-y|^{2}, \\
{[x-y][a(t, x)-a(t, y)] } & \leq-\alpha|x-y|^{2},
\end{aligned}
$$

for $t \geq t_{0}, x, y \in R^{n}$ where $\alpha, \beta$ are fixed positive numbers. Finally, let

$$
\beta \leq \frac{2 \alpha}{(2 \mu+3) n} \text { and } E\left|X_{0}\right|^{4(\mu+2)}<\infty
$$

Then there exist $h_{0}>0$ such that for any Euler scheme of the form (1.3)(1.4) with $h \in\left(0, h_{0}\right]$, defined on the infinite time-interval $\left[t_{0}, \infty\right)$ we have the following estimate:

$$
\sup _{t \geq t_{0}} E\left|X_{t}-\bar{X}_{t}\right|^{2} \leq C h
$$

where $C$ is a positive constant.

Proof. By refering to the proof of Theorem 3.1 let us consider the same augmented system (3.5) and the same function $f_{k}$ as in (3.7). We use Theorem 2.1 for the function $f_{k}$ and arrive at equality (3.10) in the same way as in the proof of Theorem 3.1. By applying our present assumptions we obtain the following recurrence relation for the mean square error $\epsilon_{k}^{2}$ :

$$
\epsilon_{k+1}^{2} \leq \epsilon_{k}^{2}-\gamma h \epsilon_{k}^{2}+\int_{0}^{h}(h-\theta) E\left\{\left(\mathcal{L}^{2} f_{k}\right)\left(t_{k}+\theta, X_{t_{k}+\theta}, W_{t_{k}+\theta}, \bar{X}_{t_{k}}, W_{t_{k}}\right)\right\} d \theta
$$

where $h>0, k \in N$ and with $\gamma=2 \alpha-\beta$ being a positive number. By applying Lemma 4.1 with $p=\mu+2$ and Lemma 4.2 we obtain

$$
\sup _{t \geq t_{0}} E\left|X_{t}\right|^{2 \mu+4} \leq C_{1}<\infty \text { and also } \sup _{k \in N} E\left|\bar{X}_{t_{k}}\right|^{2} \leq C_{2}<\infty \text {, }
$$

where $C_{1}, C_{2}$ are positive constants and $\bar{X}$ denotes the Euler scheme (1.3)-(1.4) defined for $h \in\left(0, h_{1}\right]$ ( $h_{1}$ sufficiently small). In view of (4.6), by analyzing the second iteration $\mathcal{L}^{2} f_{k}$ we write the following estimate:

$$
E\left\{\left(\mathcal{L}^{2} f_{k}\right)\left(t_{k}+\theta, X_{t_{k}+\theta}, W_{t_{k}+\theta}, \bar{X}_{t_{k}}, W_{t_{k}}\right)\right\} \leq C_{3}<\infty,
$$

for $k \in N, h \in\left(0, h_{1}\right], \theta \in[0, h]$. From (4.5) and (4.7) we derive a recurrence relation for $\epsilon_{k}^{2}$ :

$$
\begin{aligned}
\epsilon_{k+1}^{2} & \leq(1-\gamma h) \epsilon_{k}^{2}+C_{3} h^{2} \\
\epsilon_{0}^{2} & =0
\end{aligned}
$$


for $k \in N$. The above relation implies that

$$
\epsilon_{k}^{2} \leq C_{3} \frac{1-(1-\gamma h)^{k}}{\gamma h} h^{2} \leq \frac{C_{3}}{\gamma} h,
$$

for $k \in N$ and $h \in\left(0, h_{0}\right]$ where $h_{0}=\min \left(1 / \gamma, h_{1}\right)$. Finally, by noting the inequality

$$
\sup _{t \geq t_{0}} E\left|X_{t}-\bar{X}_{t}\right|^{2} \leq C_{4}\left(h+\sup _{k \in N} E\left|X_{t_{k}}-\bar{X}_{t_{k}}\right|^{2}\right)
$$

where $C_{4}$ is a positive constant, the conclusion of the theorem follows from (4.8).

Example. A simple example for applicability of the above methods is provided by the following system of stochastic differential equations:

$$
\begin{aligned}
d X_{t} & =\left[A(t) X_{t}+A_{0}(t)\right] d t+\sigma\left(t, X_{t}\right) d W_{t} \\
X_{t_{0}} & =X_{0},
\end{aligned}
$$

where $A=A(t)$ is an $n \times n$ matrix and $A_{0}=A_{0}(t)$ is an $n$-dimensional vector for any $t \geq t_{0}$. Assuming appropriate conditions for $A, A_{0}, \sigma, X_{0}$ and applying Theorem 3.1 one obtains approximate solutions of system $(4.9)-(4.10)$ on any time-interval of a finite length. In general the constant in the estimate for the mean-square error of the approximation will depend exponentially on the length of the time-interval.

Next, let us additionally assume that $A_{0}(t)=0, \sigma(t, 0)=0$ and also

$$
[A(t) x][x] \leq-\alpha|x|^{2},
$$

for $t \geq t_{0}, x \in R^{n}$, where $\alpha>0$. Then Theorem 4.1 provides us with approximate solutions of system (4.9)-(4.10) converging to the solution process $X$ uniformly on the infinite time-interval $\left[t_{0}, \infty\right)$.

\section{References}

[1] A. Friedman, "Stochastic Differential Equations and Applications", Vol 1, Academic Press, New York, 1975.

[2] I.I. Gichman, A.V. Skorochod, "Stochastic Differential Equations", Springer-Verlag, New York, 1972.

[3] G. Ladde and V. Lakshmikantham, "Random Differential Inequalities", Academic Press, New York, 1980.

[4] E.J. McShane, "Stochastic Calculus and Stochastic Models", Academic Press, New York, 1974.

[5] G.N. Mil'shtein, "Approximate integration of stochastic differential equations", Theory Prob. Appl., 19, 1974, 557-562. 
[6] G.N. Mil'shtein, "A method of second order accuracy integration of stochastic differential equations", Theory Prob. Appl., 23, 1978, 369-401.

[7] E. Platen, "An approximate method for a class of Itô processes", Litovsk. Matem. Sb., 21, 1981, 121-133.

[8] W. Rumelin, "Numerical treatment of stochastic differential equations", SIAM J. Numer. Anal., 19, 1982, 604-613. 


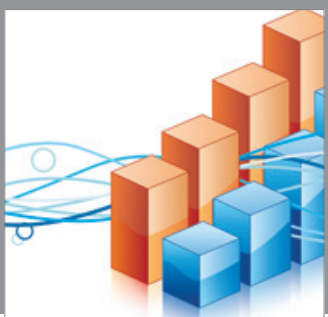

Advances in

Operations Research

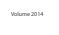

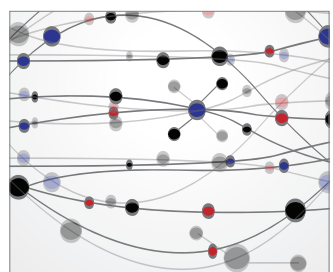

\section{The Scientific} World Journal
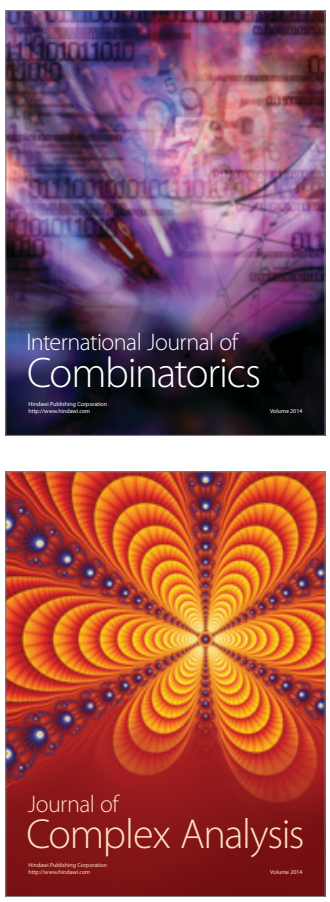

International Journal of

Mathematics and

Mathematical

Sciences
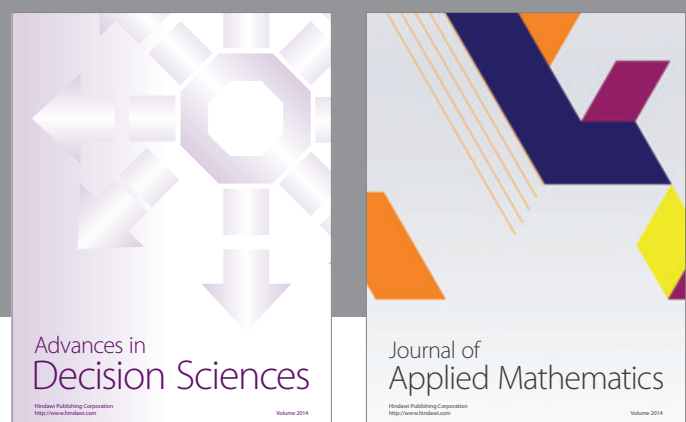

Journal of

Applied Mathematics
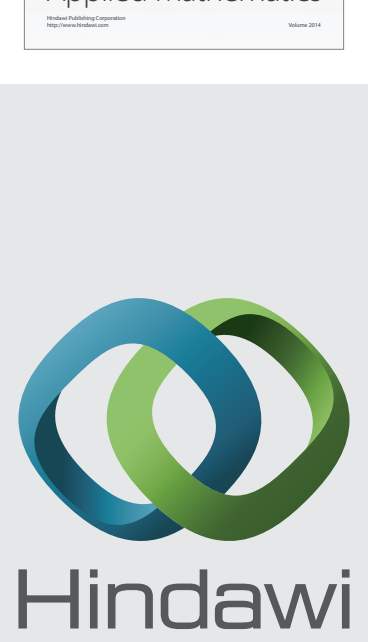

Submit your manuscripts at http://www.hindawi.com
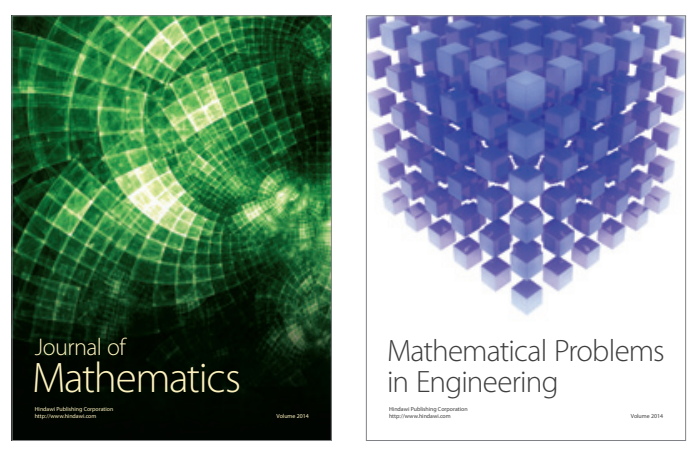

Mathematical Problems in Engineering
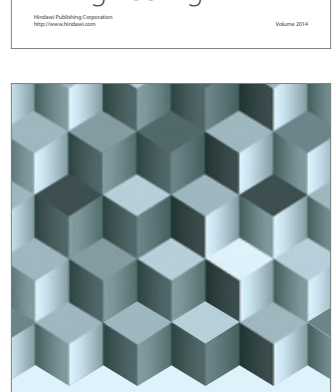

Journal of

Function Spaces
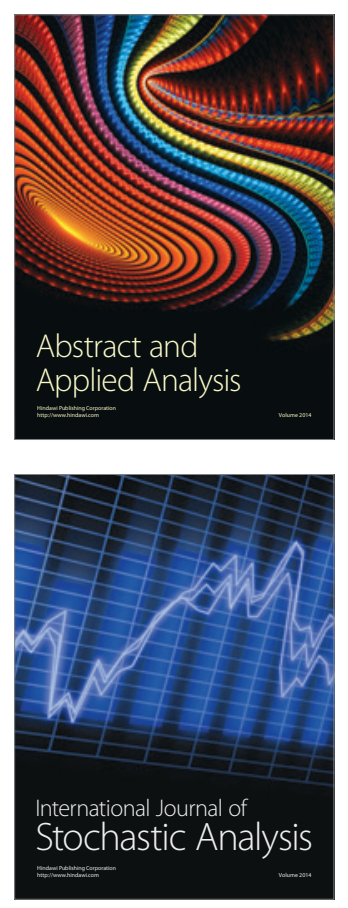

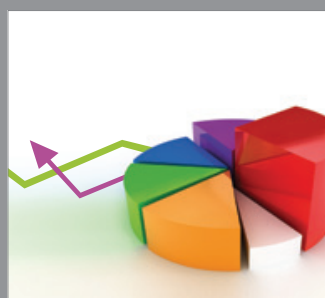

ournal of

Probability and Statistics

Promensencen
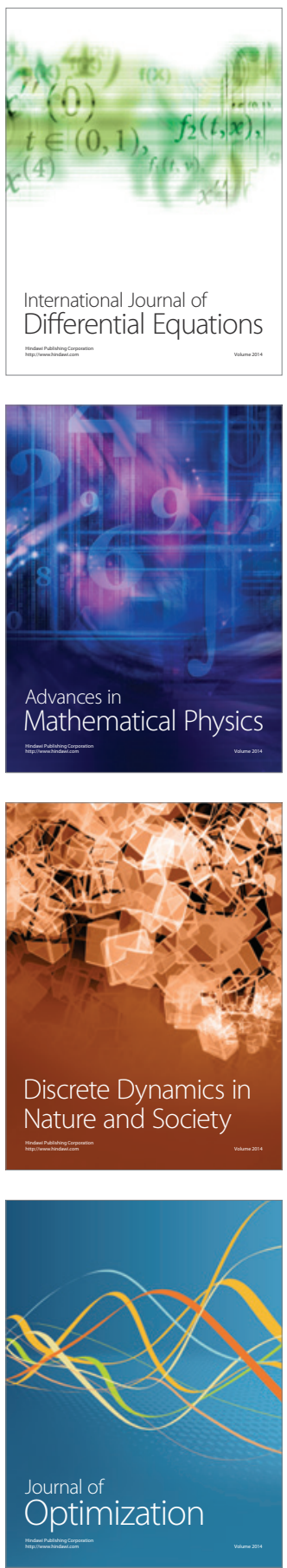